Welche Auswirkungen haben die Regelungen zum Vorteilsausgleich?

\section{Ökonomische Nutzung von genetischen Ressourcen}

\author{
Die neunte Vertragsstaatenkonferenz zum Übereinkommen \\ über die biologische Vielfalt in Bonn befasste sich auch mit \\ dem Zugang und Vorteilsausgleich für genetische Ressourcen. \\ Welche Auswirkungen haben bisherige Regelungen für Unter- \\ nehmen und vor welchem Hintergrund sind die Verhandlun- \\ gen zu bewerten? Von Karin Holm-Müller und \\ Sabine Täuber
}

V om 19. bis 30. Mai tagte in Bonn die neunte Vertragsstaatenkonferenz zum Übereinkommen über die biologische Vielfalt (Convention on Biodiversity; kurz CBD). Eines der zentralen Themen dieser Tagung sind die Regelungen zum Zugang und Vorteilsausgleich bei genetischen Ressourcen.

In der CBD wurde die Anerkennung der nationalen Souveränität der Ursprungsländer über ihre genetischen Ressourcen festgelegt, sofern die Ressourcen nicht schon vor 1992 außerhalb ihrer Territorien vorhanden waren beziehungsweise ihre Nutzung nicht durch andere Abkommen geregelt ist, wie das beispielsweise bei pflanzengenetischen Ressourcen für die Landwirtschaft der Fall ist (Rosendahl 1995). Die Regierungen der einzelnen Staaten können demnach in eigener Zuständigkeit und nach ihren innerstaatlichen Regelungen bestimmen, wer genetische Ressourcen aus der Natur oder landeseigenen Sammlungen entnehmen darf.

Dieser Zugang zu genetischen Ressourcen muss dabei unter „einvernehmlich festgelegten Bedingungen“ erfolgen, wobei eine gerechte Beteiligung der Ursprungsländer an den Vorteilen aus der Nutzung ihrer genetischen Ressourcen sicherzustellen ist. Dieses Prinzip bezeichnet man als Vorteilsausgleich. Zugang und Vorteilsausgleich werden meist zusammen behandelt und als „Access and Benefit-sharing“ (ABS) bezeichnet.

Es war erwartet worden, dass durch die Verpflichtung zum Vorteilsausgleich in den Ursprungsländern ein Eigeninte- resse am Erhalt der Natur entsteht. Ausgangspunkt war ein schon 1991 ausgehandelter Vertrag zwischen Costa Rica und dem Pharmaunternehmen Merck. Das costa-ricanische Biodiversitätsinstitut InBio erhielt damals von Merck eine Vorabzahlung von einer Million US-Dollar sowie Hilfe beim Aufbau von Forschungskapazitäten. Darüber hinaus wurde eine Gewinnbeteiligung auf alle Produkte, die direkt oder indirekt auf das erhaltene Material aufbauen, vereinbart.

\section{Positive wirtschaftliche Effekte}

Folgend auf die Verabschiedung der CBD waren die Philippinen das erste Land, das eine nationale Regelung des Zugangs zu ihren Ressourcen aufstellte. Weil sie befürchteten, von potenziellen Nutzern nicht fair und gleichberechtigt an den Vorteilen beteiligt zu werden, fiel diese Regelung recht restriktiv aus. Als Folge wurde in den Philippinen von 1996 bis 2004 nur ein Vertrag zur kommerziellen Nutzung von genetischen Ressourcen geschlossen (Richerzhagen 2007). Die monetären Erträge aus diesem Vertrag sind bisher sehr bescheiden (GDI 2003).

Als positives Beispiel für ein nationales ABS-Konzept kann dagegen Costa Rica gelten. Hier hat sich InBio zu einem führenden Partner für Unternehmen entwickelt, die genetische Ressourcen verwenden, und mit solchen bereits mehrere Verträge über die Bereitstellung von genetischem Material unterzeichnet. Ri- cherzhagen spricht von 24 wichtigen kommerziellen und akademischen Vereinbarungen, die in Costa Rica von 1991 bis 2004 geschlossen wurden (Richerzhagen 2007). Durch die Kooperationen haben sich außerdem mehrere kleinere Unternehmen in Costa Rica entwickeln können, die biologisches Material für den heimischen Markt verwenden (Richerzhagen 2005). Lizenzgebühren sind allerdings bis 2004 noch nicht geflossen, sodass die Einnahmen aus ABS-Verträgen im Vergleich $\mathrm{zu}$ anderen Landnutzungsformen wie Forstwirtschaft und Tourismus auch dort bisher bescheiden sind (Richerzhagen 2005).

In einer Übersicht von 2003 beschreibt das GDI fünf bekannt gewordene Beispiele für ABS-Abkommen und schließt daraus, dass angesichts der geringen Geldtransfers aus Vorabzahlungen und der vereinbarten Gewinnbeteiligungen, die immer zwischen einem und fünf Prozent liegen, die finanziellen Vorteile aus den ABS-Abkommen in der Regel gering ausfallen. Die Vorteile reichen weder aus, um für die Bereitstellerländer die Opportunitätskosten eines Biodiversitätserhalts zu kompensieren, noch um die ökonomischen Möglichkeiten der Armen mehr als marginal zu verbessern (GDI 2003).

Wenn man bedenkt, dass die Entwicklung von Produkten in der Pharmaindustrie im Durchschnitt 15 Jahre dauert, ist es allerdings durchaus möglich, dass in Zukunft weltweit deutlich mehr Gelder aus Gewinnbeteiligungen bereits bestehender ABS-Verträge in die Bereitstellerländer fließen als bisher (Ten Kate 1999). Andererseits gibt es bis heute auch kaum positive Beispiele für Ursprungsländer, die ihnen signalisieren, dass sich aus dem Naturschutz durch ABS Gewinne machen ließen oder auch nur, dass sie an den Gewinnen der Nutzerländer aus der Biodiversitätsnutzung wirklich fair beteiligt werden.

\section{Schwierige Abstimmungs- prozesse}

Gleichzeitig gibt es in den Nutzerindustrien Bestrebungen, auf die Nutzung von genetischen Ressourcen, die unter 
die CBD fallen, so weit wie möglich zu verzichten. Dies wird unter anderem mit zeitraubenden und unkalkulierbaren Prozessen für ABS-Abkommen begründet. Ein Beispiel hierfür gibt das 2003 gestartete und vom BMBF geförderte Projekt Pro-Benefit, in dem für ein bestimmtes Gebiet in Ecuador ein Bio-ProspektionsAbkommen geschlossen werden sollte. In dem Projekt wurde erfolglos mehr als vier Jahre lang mit über 40 potenziellen Gruppen verhandelt, um die im Rahmen von ABS geforderte vorherige informierte $\mathrm{Zu}$ stimmung der Betroffenen zu erhalten. (Ploetz 2008).

Auch eineinhalb Jahrzehnte nach dem Inkrafttreten der Konvention über die biologische Vielfalt muss die Umsetzung des Zugangs und Vorteilsausgleichs als unbefriedigend gelten. Als Folge haben sich die Vertragsstaaten zum Ziel gesetzt, bis 2010 ein internationales Regime zur Durchsetzung des Zugangs und Vorteilsausgleichs $\mathrm{zu}$ verhandeln. In einer Arbeitsgruppe wird seitdem beispielsweise die Einführung unterschiedlicher Formen von Zertifikaten diskutiert, mit deren Hilfe die Einhaltung der Rechte der Ursprungsländer abgesichert werden sollen. Der Nutzer würde im Falle der Einführung eines solchen Zertifikats verpflichtet, dieses an noch zu bestimmenden Stellen im Nutzungsprozess, zum Beispiel der Patentanmeldung, vorzulegen (Dross 2005).

\section{Modelle zur Vereinfachung}

Gleichzeitig gibt es auch Überlegungen zu Modellverträgen oder Modellklauseln für ABS-Abkommen, von denen man sich eine erhebliche Verringerung von Transaktionskosten verspricht. Seit Kurzem gibt es Initiativen wie Science Commons, die Systeme zum Austausch von genetischen Ressourcen unter Anwendung von Modellklauseln entwickeln oder untersuchen, für welche Arten von Ressourcen oder Verwendungen solche Lösungen Anwendung finden könnten (Täuber 2008).

Während die Ursprungsländer Mindestbedingungen für einen fairen Vorteilsausgleich in einem internationalen Regime festlegen wollen, geht es den Nut- zerländern in erster Linie um einen vereinfachten Zugang. Um diese unterschiedlichen Interessen zu versöhnen, wird es eventuell notwendig sein, mehrere Ziele, wie beispielsweise die Einrichtung von Schutzgebieten, bei den Verhandlungen zu berücksichtigen. Dies erweitert die Möglichkeiten, für Zugeständnisse auf einem Feld Vorteile in anderen Bereichen zu erzielen. Man darf gespannt sein, ob es so gelingt, den wichtigen Bereich des Zugangs und Vorteilsausgleichs bis 2010 erfolgreich zu regeln.

\section{Literatur}

Dross, M. / Wolff, F.: New Elements of the International Regime on Access and Benefit- Sharing of Genetic Resources. Bonn 2005.

GDI - German Development Institute: Access and Benefit-Sharing (ABS): An Instrument for Poverty Alleviation. Bonn 2003.

Ploetz, C.: Lessons learned from an ABS project. Presentation at ABS WG 6. Geneva 2008. Internet: http://www.probenefit.de/files/Vortrag_CBD.ABS_Genf_0801_engl_ploetz.pdf (12.03.2008).

Richerzhagen, C.: Effectiveness and Perspectives of Access and Benefit-sharing Regimes in the Convention on Biological Diversity. Dissertation Universität Bonn 2007.

Richerzhagen, C. / Holm-Müller, K.: The effectiveness of access and benefit-sharing in Costa Rica. In: Ecological Economics 53, 2005. S. 445-460.

Rosendahl, G. K.: The Convention on Biological Diversity. In: Bergesen, O. H. / Parmann, G. / Thommessen, O. B. (Hrsg.): Green globe yearbook of international co-operation on environment and development. Oxford 1995. S. 69-81.

Science Commons: Biological Materials Transfer Project. http://sciencecommons.org/projects/licensing/ (12.03.2008).

Täuber, S. / Holm-Müller, K.: Economic analysis of various design options for an international regime on access and benefit-sharing in the framework of the CBD.

http://www.bioecon.ucl.ac.uk/02r_3agri.html (25.03.2008).

Ten Kate, K. / Laird, S.: The commercial use of biodiversity. London 1999.

I AUTORINNEN + KONTAKT

Karin Holm-Müller ist Leiterin und Sabine Täuber ist wissenschaftliche Mitarbeiterin an der Professur für Ressourcen- und Umwelt ökonomik der Universität Bonn.

Institut für Lebensmittel- und Ressourcenökonomik der Universität Bonn, Abteilung Ressourcenund Umweltökonomik, Nussallee 21, 53113 Bonn. E-Mail: karin.holm-mueller@ilr.uni-bonn.de; sabine.taeuber@ilr.uni-bonn.de

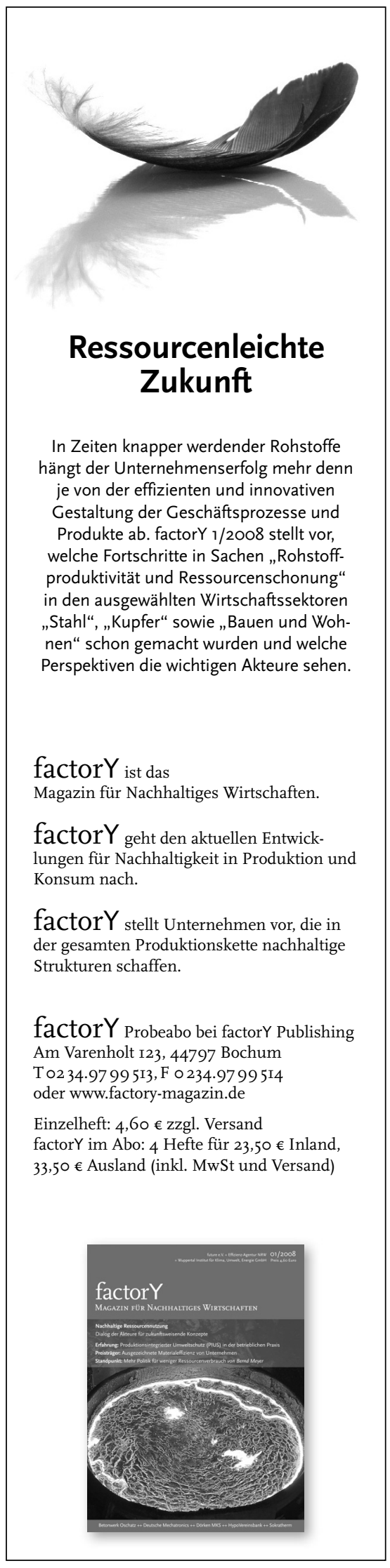


(c) 20I0 Authors; licensee IÖW and oekom verlag. This is an article distributed under the terms of the Creative Commons Attribution Non-Commercial No Derivates License (http://creativecommons.org/licenses/by-nc-nd/3.o/), which permits unrestricted use, distribution, and reproduction in any medium, provided the original work is properly cited. 